\title{
Corrigendum: Joint analysis is more efficient than replication-based analysis for two-stage genome-wide association studies
}

Andrew D Skol, Laura J Scott, Gonçalo R Abecasis \& Michael Boehnke

Nat. Genet. 38, 209-213 (2006).

In Table 1 of the versions of this article initially published online and in print, the significance thresholds for $C_{2}$ were incorrect, and the significance thresholds for $C_{\text {joint }}$ in the case of $\pi_{\text {samples }}=0.20$ were incorrect. The error has been corrected in the HTML and PDF versions of the article. This correction has been appended to the PDF version.

Table 1 Significance thresholds and power of joint analysis for two-stage genome-wide association designs

\begin{tabular}{|c|c|c|c|c|c|c|c|c|c|c|c|}
\hline \multirow[b]{3}{*}{$\pi_{\text {samples }}$} & \multirow[b]{3}{*}{$\pi_{\text {markers }}$} & \multirow{3}{*}{$\begin{array}{l}\text { Proportion of } \\
\text { genotypes }^{\mathrm{a}}\end{array}$} & & & & \multicolumn{6}{|c|}{ Power } \\
\hline & & & \multicolumn{3}{|c|}{ Significance threshold } & \multicolumn{2}{|c|}{$\mathrm{GRR}=1.30$} & \multicolumn{2}{|c|}{$\mathrm{GRR}=1.35$} & \multicolumn{2}{|c|}{$\mathrm{GRR}=1.40$} \\
\hline & & & $C_{1}$ & $C_{2}$ & $C_{\text {joint }}$ & Joint & Rep & Joint & Rep & Joint & Rep \\
\hline 1.0 & 0 & 1.00 & - & - & 5.23 & 0.26 & - & 0.51 & - & 0.75 & - \\
\hline \multirow[t]{3}{*}{0.50} & 0.10 & 0.55 & 1.64 & 4.65 & 5.23 & 0.26 & 0.08 & 0.51 & 0.17 & 0.75 & 0.31 \\
\hline & 0.05 & 0.53 & 1.96 & 4.50 & 5.23 & 0.26 & 0.09 & 0.51 & 0.21 & 0.75 & 0.36 \\
\hline & 0.01 & 0.51 & 2.58 & 4.15 & 5.23 & 0.26 & 0.14 & 0.50 & 0.29 & 0.74 & 0.48 \\
\hline \multirow[t]{3}{*}{0.40} & 0.10 & 0.46 & 1.64 & 4.65 & 5.23 & 0.26 & 0.12 & 0.51 & 0.27 & 0.75 & 0.46 \\
\hline & 0.05 & 0.43 & 1.96 & 4.50 & 5.23 & 0.26 & 0.14 & 0.50 & 0.30 & 0.74 & 0.51 \\
\hline & 0.01 & 0.41 & 2.58 & 4.15 & 5.20 & 0.24 & 0.17 & 0.48 & 0.36 & 0.71 & 0.58 \\
\hline \multirow[t]{3}{*}{0.30} & 0.10 & 0.37 & 1.64 & 4.65 & 5.22 & 0.25 & 0.17 & 0.50 & 0.36 & 0.73 & 0.58 \\
\hline & 0.05 & 0.34 & 1.96 & 4.50 & 5.21 & 0.24 & 0.18 & 0.48 & 0.37 & 0.72 & 0.60 \\
\hline & 0.01 & 0.31 & 2.58 & 4.15 & 5.16 & 0.21 & 0.18 & 0.42 & 0.37 & 0.64 & 0.58 \\
\hline \multirow[t]{3}{*}{0.20} & 0.10 & 0.28 & 1.64 & 4.65 & 5.19 & 0.23 & 0.19 & 0.46 & 0.39 & 0.68 & 0.62 \\
\hline & 0.05 & 0.24 & 1.96 & 4.50 & 5.16 & 0.21 & 0.18 & 0.42 & 0.38 & 0.63 & 0.59 \\
\hline & 0.01 & 0.21 & 2.58 & 4.15 & 5.06 & 0.15 & 0.14 & 0.31 & 0.29 & 0.47 & 0.46 \\
\hline
\end{tabular}

Shown is analysis of 1,000 cases and 1,000 controls, $M=300,000$ markers, genome-wide significance level $\alpha$ genome $=0.05$, multiplicative model, control risk allele frequency $=0.40$ and prevalence $=0.10$.

a(Number of genotypes required for two-stage design) / (number of genotypes required when all markers are genotyped on all samples). 